\title{
Development of Integrated Analysis System of Medical and LTCI Claim Data in Japan
}

\author{
Shinya Matsuda'), Kenji Fujimori'2) \\ ${ }^{1)}$ Department of Preventive Medicine and Community Health, School of Medicine, University of Occupational \\ and Environmental Health \\ 2)Hokkaido University Hospital
}

\begin{abstract}
As the Japanese health services are provided under the different scheme, it is relatively difficult to analyze the data by an integrated way. In order to ameliorate this situation, we have developed a model system for integrated analysis of health insurance claim, the long term care insurance claim and the disease management data. We used the National Health Insurance claim data (medical services and pharmaceuticals), the LTCI claim data and the lab-data of disease management program of a local town of Fukuoka prefecture. The data is one month's data September 2009. We have developed a SQL program to combine above mentioned data by the individual client basis. Using a set of combined data, we have examined total expenditures stratified by age category, diagnosis, and existence of obesity. There are several interesting results obtained. Female dominantly used the LTCI services after 70 yr old but male always used health insurance covered services compared with those covered by the LTCI scheme. For the health insurance expenditures, neurological disorders were important for female and the respiratory disorders and the disorder of digestive system, hepato-billiary system and pancreas were dominant for male. The present study clarified the usefulness of integrated evaluating system of claim data. However there are some problems to be solved; creation of unique ID, coding of multiple diagnosis, and enrolment of nationally defined format of each claim.
\end{abstract}

Key words: e-claim, unique ID, health insurance, LTCI, disease management, health expenditures

\section{Introduction}

Along with the ageing of society, the social security expenditures continue to increase in Japan. The government, especially the Ministry of Finance, is very anxious about its sustainability. According to the international comparative study by OECD, Japan uses a relatively small part of GDP for medical services $(9.0 \% \text { in } 2008)^{1)}$. Therefore, the medical professionals demand more resource allocation for the health

Received: September 29, 2010

Accepted: July 20, 2011

Correspondence: S. Matsuda, Department of Preventive Medicine and Community Health, School of Medicine, University of Occupational and Environmental Health, 1-1 Iseigaoka, Yahatanishi-ku, Kitakyushu, Fukuoka 807-8555, Japan e-mail: smatsuda@med.uoeh-u.ac.jp sector, in order to avoid the catastrophic situation ${ }^{2)}$. There is a tough debate between the two sides.

The Prime Minster, Naoto Kan, has proposed "the third way" that intends to increase the investment to medical and Long Term Care Insurance (LTCI) services in order to realize the strong economy and the reliable social security system simultaneously ${ }^{3)}$.

In fact, the previous Input-Output studies have clarified that the investment for health sector could have a similar or better effect on general economy and job creation compared with the investment for civil construction sector ${ }^{4}$. In this meaning, the authors would like to positively evaluate the Kan's third way approach. However, the history of investment by governments teaches us the unreliability of their projects. It is very easy to list up the un-succeeded projects resulted in the immense deficit that have been orga- 


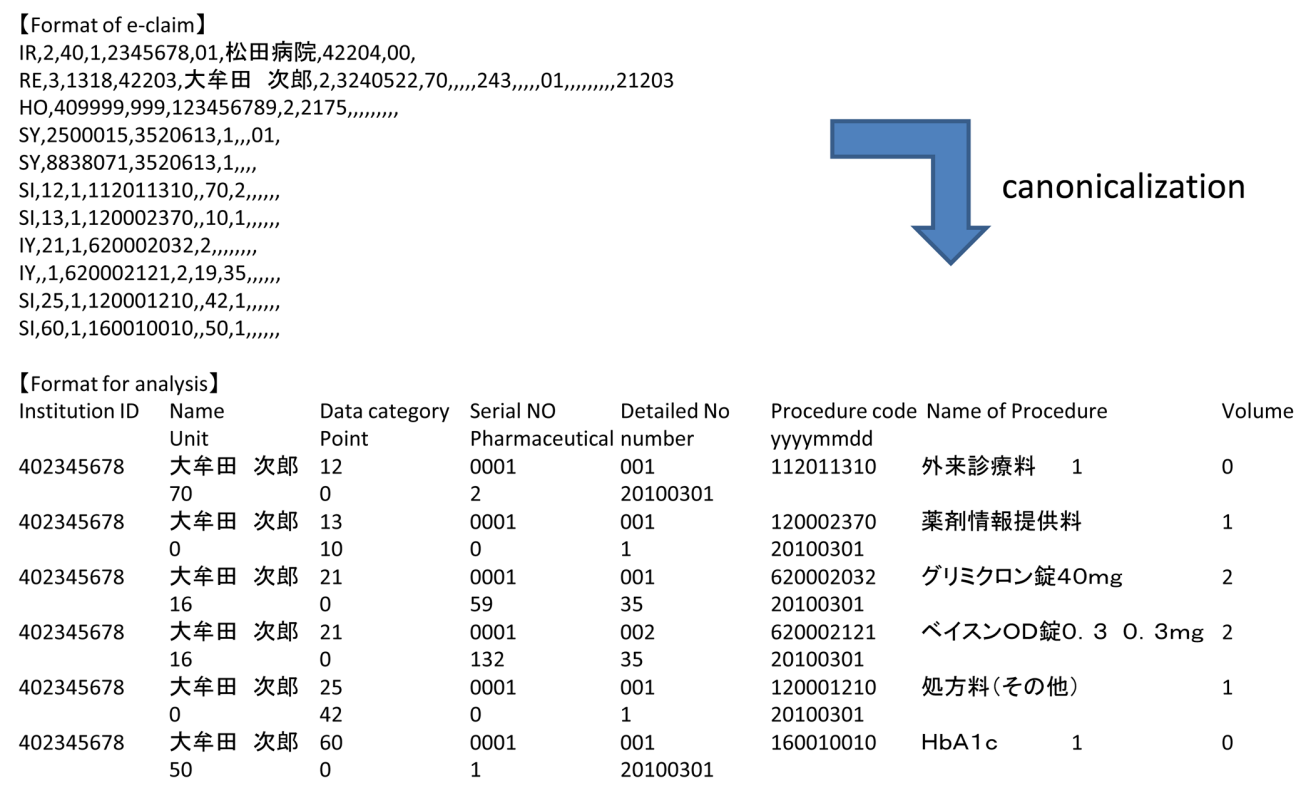

Figure 1 Format of e-claim and data format for analysis.

nized by the central and local governments, i.e., under used airports and highways.

In order to implement a program properly, it is indispensible to prepare the monitoring and evaluation system. In the case of health policy, the government must have an evaluation system under the PDCA (PlanDo-Check-Action) cycle concept. Although the Japanese insurance scheme has very useful information, that is the claim data, its use has not been developed. In 2008, the Ministry of Health, Labour and Welfare (MHLW) has introduced the disease management program targeting diabetes mellitus. Although the government explains that this program would be effective to control the increase of medical expenditures, there has no previous evidence to support this hypothesis. In order to examine this hypothesis, there must be a system that makes it possible to evaluate its effect. As it is inappropriate to evaluate the effectiveness of preventive activity within a short term, it is indispensible to implement the life-long evaluation system. This requires to combine the information of disease management program with medical and LTCI claim data.

Currently, these data are treated under the different system with different formats. Considering the financial limitation that our social security system faces, it is an urgent task to construct an integrated evaluation system in order to implement a truly effective health policy with the correct priority setting.

For this purpose, the authors have tried to con- struct an integrated evaluation system of medical, LTCI and disease management program using the data set of a local insurer of Fukuoka prefecture. According to the present results, we tried to summarize the critical points in order to generalize the constructed evaluation system.

\section{Data and Methods}

\section{Data}

We used the National Health Insurance claim data (medical services and pharmaceuticals), the LTCI claim data and the lab-data of disease management program of a local town of Fukuoka prefecture. The data is one month's data of September 2009.

The use of data was approved by the ethical committee of the town's assembly in September 2009.

\section{Methods}

The upper chart of Figure 1 shows the format of current e-claim. This format is not canonicalized, so difficult to be processed. We developed a SQL (structured query language) program to canonicalize the data format like as the under chart of Figure 1. In this way we have constructed a set of tables for further analyses as shown in Figure 2. As each claim data has its proper ID number, we asked to the town office to create the unique ID for each insured by the way we have defined.

For the diagnosis, we used the first diagnosis that 


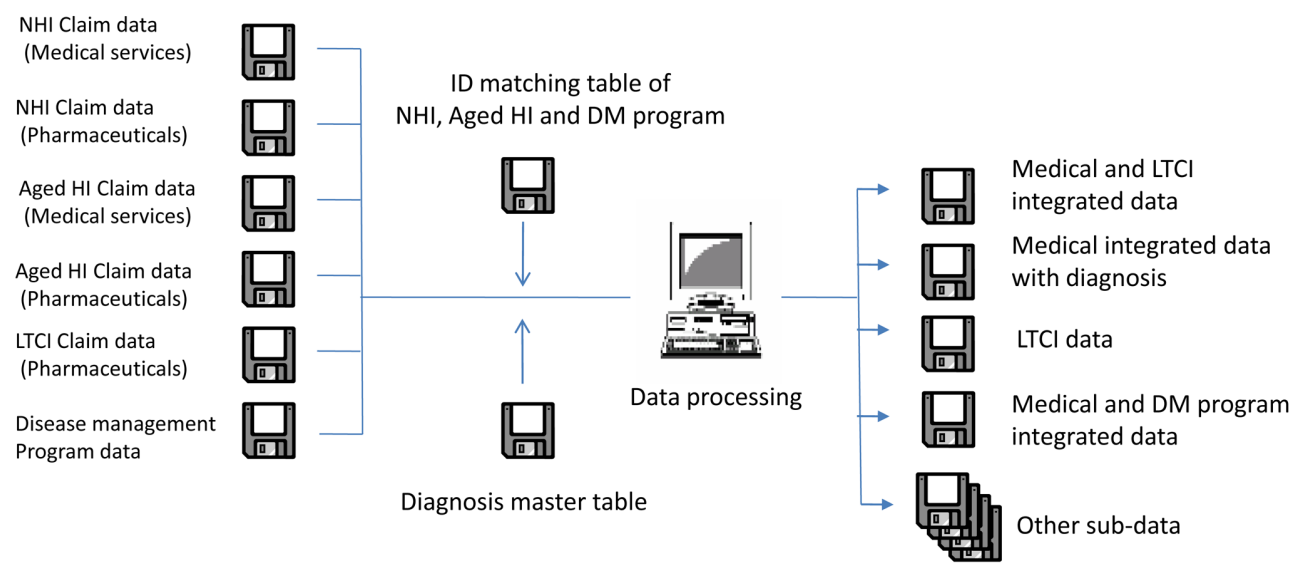

Figure 2 Total health expenditures (K town, Fukuoka Prefecture, September 2009).

Table 1 Major Diagnosis Categories

\begin{tabular}{ll}
\hline MDC & \\
\hline 01 & Diseases and disorders of the Nervous system \\
02 & Diseases and disorders of the Eye \\
03 & Diseases and disorders of the Ear, Nose, Mouth and Throat \\
04 & Diseases and disorders of the Respiratory system \\
05 & Diseases and disorders of the Circulatory system \\
06 & Diseases and disorders of the Digestive and Hepato-billiary system and Pancreas \\
07 & Diseases and disorders of the Muscloskeltal system and Connective tissues \\
08 & Diseases and disorders of the Skin and sub-cutaneous tissue \\
09 & Diseases and disorders of the Breast \\
10 & Endocrine, Nutritional and Metabolic Diseases and disorders \\
11 & Diseases and disorders of the Kidney and Urinary Tract and the Male reproductive system \\
12 & Diseases and disorders of the Female reproductive system \\
13 & Diseases and disorders of the Blood and Blood Forming Organs and Immunological disorders \\
14 & Newborn and Neonates with conditions originating in the perinatal period \\
15 & Pediatric diseases and disorders \\
16 & Injuries, Poisoning, Toxic effects of drugs and Burns \\
17 & Psychiatric diseases and disorders \\
18 & Others \\
99 & Unknown
\end{tabular}

was registered in each e-claim. The diagnosis was converted to the corresponding ICD code and then Major Diagnosis Categories (MDC) of the Japanese Casemix system, so called DPC (Diagnosis procedure combination: Table 1).

The present project was approved by the ethic committee of UOEH and the local government.

\section{Results}

The current analysis was based on the only one month's data, so there is limitation for validity of sci- entific evaluation. Kept this limitation in mind, we describe the results in the following section.

Figure 3 shows the sum of expenditures stratified by health insurance and LTCI. The expenditures by health insurance are dominant for all age categories in the case of male even though the percentage covered by LTCI increased after $70 \mathrm{yr}$ old. Female used more LTCI coverage than male and LTCI expenditures were dominant after $70 \mathrm{yr}$ old.

Figure 4 showed the health expenditures stratified by MDC and sex. It is interesting that in-patient expenditure of MDC17 were very high for both sexes. There 


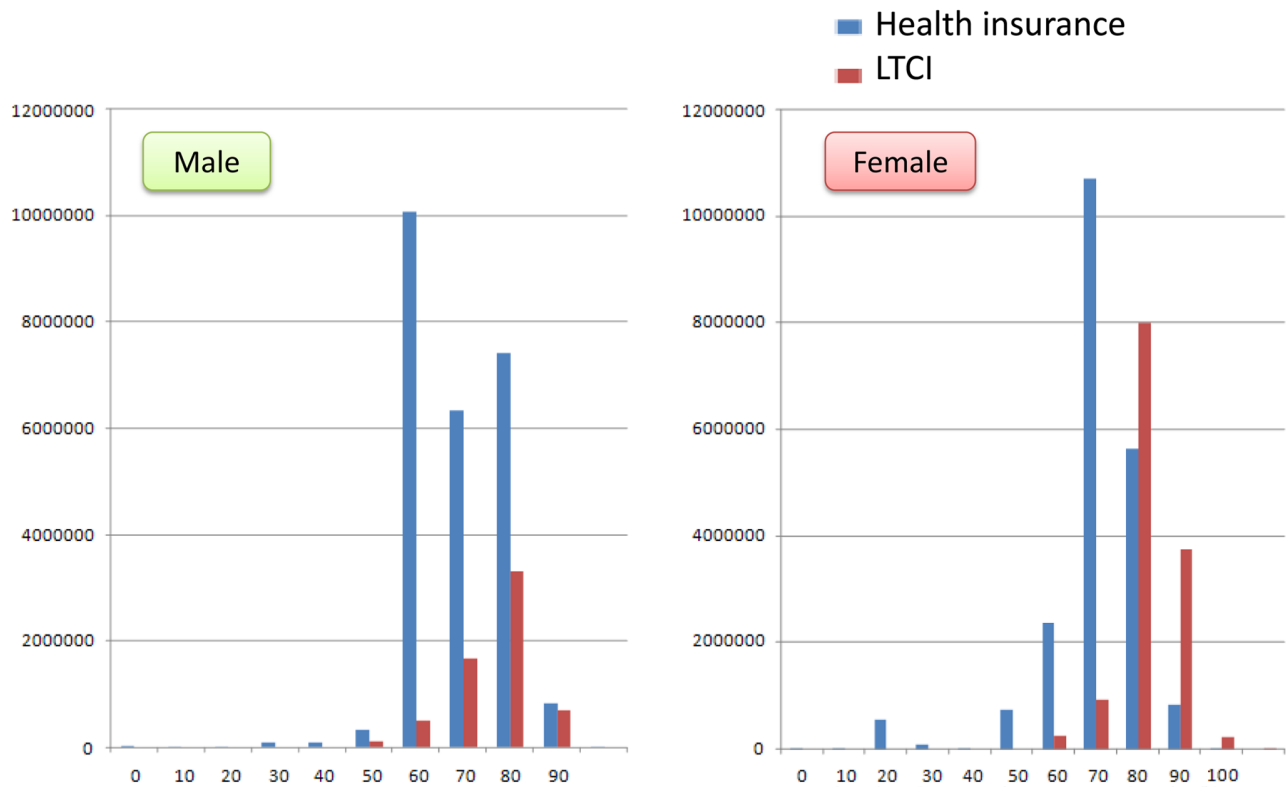

Figure 3 Total health expenditures (K town, Fukuoka Prefecture, September 2009).

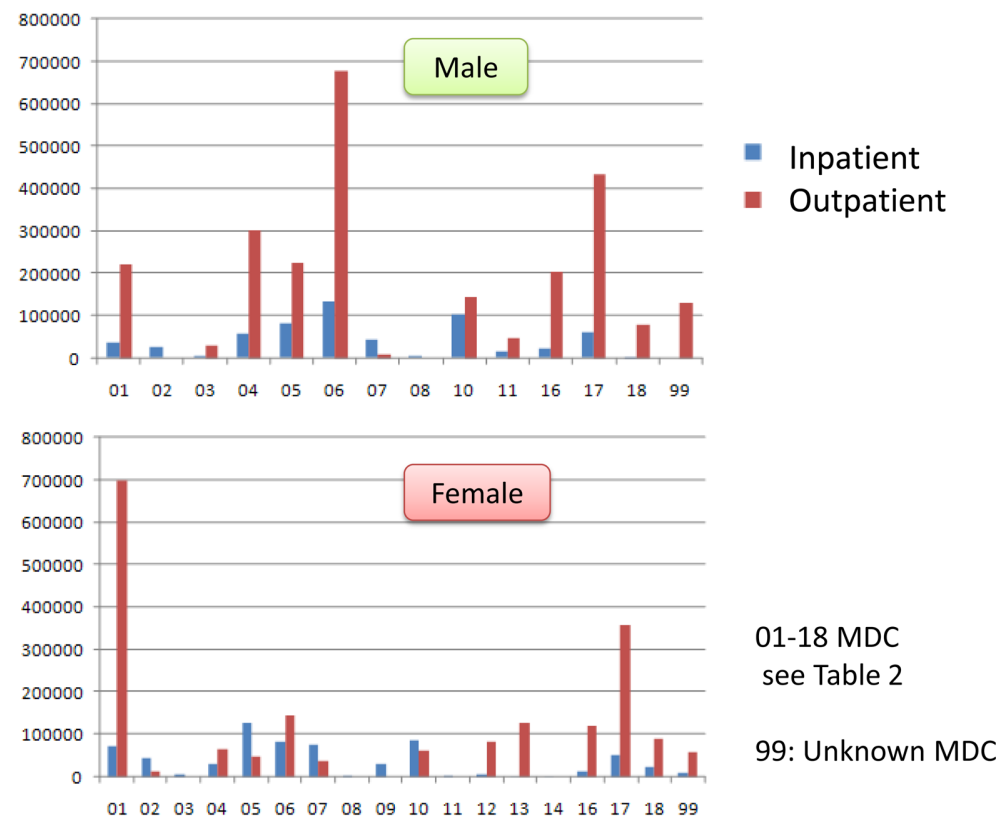

Figure 4 Total health expenditures stratified by MDC

(K town, Fukuoka Prefecture, September 2009).

are interesting sex differences for the expenditures. In the case of male, the in-patient expenditures are high for MDC04 (respiratory disorders), MDC05 (Disorder of circulatory system) and MDC06 (Disorder of digestive system, hepato-billiary system and pancreas) in the case of male. For female, the expenditures for MDC01 (neurological disorder) are high.

Figure 5 shows number of patients and per capita average expenditures for diabetes stratified by in- and out-patient services, and sex. The number of diabetic patient increases after $40 \mathrm{yr}$ old, especially after $60 \mathrm{yr}$ old. Correspondingly, per capita health expenditures drastically increases after $60 \mathrm{yr}$ old, especially for inpatient services.

Table 2 shows the medical expenditures stratified by obesity level. The higher expenditures were observed for obese female of $50 \mathrm{yr}$ old and obese male of 60 and $70 \mathrm{yr}$ old. 

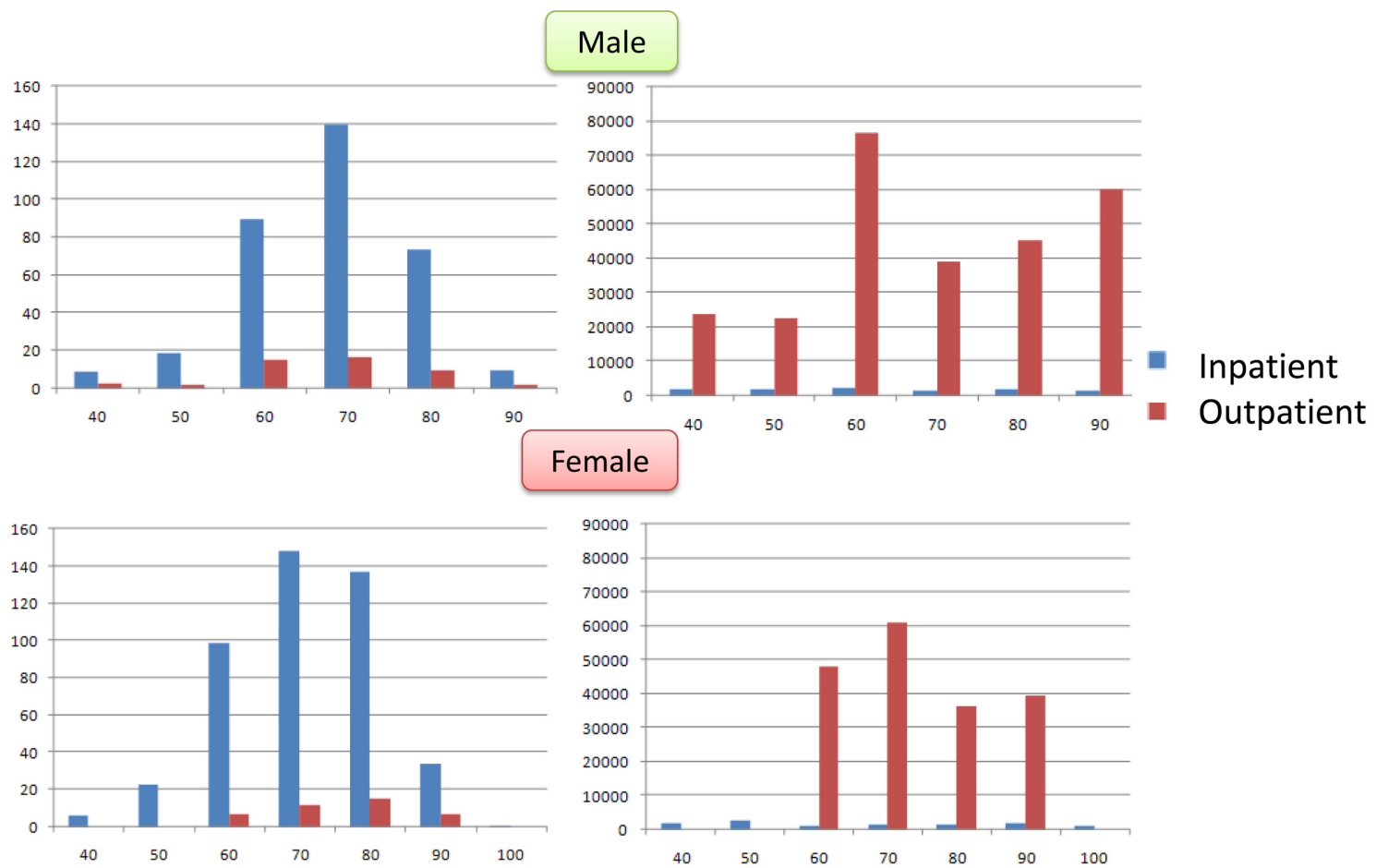

Figure 5 Number and average health expenditures of diabetic patients (K town, Fukuoka Prefecture, September 2009).

Table 2 Medical expenditures stratified by obesity level (K town, Fukuoka Prefecture, September 2009)

\begin{tabular}{|c|c|c|c|c|c|c|c|c|}
\hline & \multicolumn{4}{|c|}{ Female } & \multicolumn{4}{|c|}{ Male } \\
\hline & $\begin{array}{l}50-59 \\
\text { yr old }\end{array}$ & $\begin{array}{l}60-69 \\
\text { yr old }\end{array}$ & $\begin{array}{l}70-74 \\
\text { yr old }\end{array}$ & $\begin{array}{c}\text { Female } \\
\text { total }\end{array}$ & $\begin{array}{l}50-59 \\
\text { yr old }\end{array}$ & $\begin{array}{l}60-69 \\
\text { yr old }\end{array}$ & $\begin{array}{l}70-74 \\
\text { yr old }\end{array}$ & $\begin{array}{l}\text { Male } \\
\text { total }\end{array}$ \\
\hline \multicolumn{9}{|c|}{ Number of cases } \\
\hline Not obese & 4 & 24 & 19 & 47 & 1 & 11 & 12 & 24 \\
\hline Obese & 1 & 7 & 6 & 14 & 0 & 90 & 8 & 98 \\
\hline Total & 5 & 31 & 25 & 61 & 1 & 101 & 20 & 122 \\
\hline \multicolumn{9}{|c|}{ Average health expenditures } \\
\hline Not obese & 16,853 & 37,123 & 28,267 & 31,817 & 25,290 & 17,345 & 23,286 & 20,646 \\
\hline Obese & 27,830 & 19,661 & 27,490 & 23,600 & & 273,267 & 28,596 & 158,128 \\
\hline Total & 19,048 & 33,180 & 28,080 & 29,931 & 25,290 & 132,510 & 25,410 & 77,651 \\
\hline \multicolumn{9}{|c|}{ Total health expenditures } \\
\hline Not obese & 67,410 & 890,640 & 537,070 & $1,495,420$ & 25,290 & 190,790 & 279,430 & 495,510 \\
\hline Obese & 27,830 & 137,630 & 164,940 & 330,400 & & $2,459,400$ & 228,770 & $2,688,170$ \\
\hline Total & 95,240 & $1,028,270$ & 702,010 & $1,825,820$ & 25,290 & $2,650,190$ & 508,200 & $3,183,680$ \\
\hline
\end{tabular}

\section{Discussion}

The present study has clarified the feasibility of constructing an integrated information system that makes it possible to analyze the medical and LTCI claim data and disease management data by an individual client level. As the format of e-claim is standardized for each public program, it is relatively easy to construct a semi-automatic analyzing system by SQL programming. However, there are several problems to be solved in order to generalize our system as following;

\section{Preparation of unique ID}

As Japan does not implement the social security 
number system, each citizen has different ID numbers for different insurance systems. Therefore, it is necessary to combine different ID numbers in order to construct a unique ID. The ID numbers are allocated to each individual for the LTCI and the disease management programs, respectively. However, in the case of National Health Insurance (NHI), the ID number is allocated to each household, not to individual. Therefore a new ID number must be created for each member of the same household by using birth date, sex, and name. This procedure requires a lot of work. Recently similar projects have been planned by other organizations. It is expected that they will also face the same problem. If each organization creates the unique ID by its own way, there will be a kind of chaos. This situation will cause many problems in future. Therefore it is very important for the government to define the standard format of unique ID.

\section{Respect of national standard for e-claim processing}

One of the most difficult problems to be solved that we experienced during the research was differences in the formats of data. Even though the government defined the official format of e-claim, not all institutions respected this requirement. For example, the ID numbers were coded by 2 bite characteristics in some cases and by 1 bite characteristics in other cases. In this study we hashed the original information for the privacy issues, therefore the difference of bite caused a difficult situation where the same person was treated as another person between the different claims of the same person. In fact MHLW clearly defined the format, but some IT vendors do not follow this definition. The MHLW must enforce the official order all out. This would need some financial incentives or penalties.

\section{Coding of diagnosis}

As the Japanese payment system has long been based on the fee-for-services principle, the health services providers have been requested to list up all the diagnoses that correspond to the provided procedures. As a result, it is very common that a claim data contains more than 4 diagnoses. In the present study, there was a claim that contained 81 diagnoses! Under the current rule of listing up diagnoses, it is not necessary to clarify the association between diagnoses and procedures. This situation makes it difficult to estimate the expenditures for each diagnosis. In order to clarify the effectiveness of disease management program targeting the metabolic syndrome, it is indispensible to be able to analyze the data by diagnosis basis.

\section{* Conclusion}

Although the generalization of e-claim has long been essential in order to formulate a more reasonable health policy, it has not been materialized up to now. One of the most important obstacles seems to be an opposition from the medical professionals. Although they understand its need very well, they are reluctant because they are afraid of too much control by the government.

Another obstacle may be a hesitation of IT vendors. They have constructed the e-claim processing system and Electronic Health Record (EHR) by their own way of format. If the format is standardized, they may lose their already kept clients and may be faced to the declining prices and then the reduced profit.

Considering the immense volume of deficit that central and local governments face, there is no choice to reduce unnecessary cost. It seems reasonable to shift the indirect cost to the direct cost for services. In this meaning, the standardization of health related information is indispensible. Currently, there is a tough debate about the introduction of social security number for each citizen. Most of health professionals are against its introduction by questioning about privacy problem. However, this social security system has been successfully introduced among the Nordic countries. It is the time when we have to discuss this issue from the more positive way of thinking.

\section{References}

1) Japan Medical Association: Ground design 2009 for health system, February 2009.

2) Prime minister Naoto Kan's general policy speech, 11 June 2010, http://www.kantei.go.jp/jp/kan/statement/201006/11syosin.html (access 24 June 2010).

3) Matsuda S, Murata H, Funatani F: Input-output analysis of investment in health sector of Kitakyushu city. Japanese Journal of Health Economics and Policy 4: 51-70 (1997).

4) Institute for Health Economics and Policy: Report on input-output analysis of investment in medical and welfare sectors. Tokyo: IHEP, 2004. 